\title{
Análise das zonas de perfil conceitual dos estudantes em episódios de ensino de Físico-Química mediados pelo computador.
}

Rodrigo Garrett da Costa, PGIE/UFRGS, IFF Campos Centro/RJ, garrett@iff.edu.br Liliana Maria Passerino, PGIE/UFRGS, liliana@ pgie.ufrgs.br

Milton Antonio Zaro, PGIE/UFRGS, zaro@ufrgs.br

\section{Resumo}

O estudo apresenta uma análise das principais contribuições da Teoria Sócio-histórica para a formação de conceitos em Físico-Química. Serão discutidos, com base em leituras de alguns pesquisadores neovygotskyanos, os pressupostos da teoria de Vygotsky e seus desdobramentos para o nosso campo de estudo, a Química. As diferentes concepções sobre o processo de formação de conceitos científicos serão analisadas, tomando-se como base suas inter-relações com os conceitos de senso comum. Em seguida, será proposta uma sequência de episódios de ensino mediados pelo computador, no eixo temático "termodinâmica química", para a análise das zonas de perfil conceitual dos estudantes.

Palavras-chave: Teoria Sócio-histórica, Aprendizagem, Conceitos, Físico-Química, Tecnologias.

\begin{abstract}
This work goal to analyze contributions of the Socio-historical Theory to understand scientific concepts development about Physical Chemistry Area. In this work we will discuss, based on some neo-vygotskian research, the impact that theory on our study field, in this case the Chemistry. After that, we will analyze the different conceptions about the process of development of scientific concepts, based on their interrelationships with the "common sense" concepts. Finally, we propose an episode in education mediated by computer, the main theme "chemical thermodynamics", for the analysis of the areas of conceptual profile of students.
\end{abstract}

Keywords: Socio-historical Theory, Learning, Concepts, Physical Chemistry, Technology.

\section{Introdução}

O ensino de ciências depara-se com o desafio de desvendar o universo diante dos olhos dos alunos. Esse processo, chamado por Chassot (2003) de "alfabetização cientifica", consiste em compreendermos o que está ao nosso redor por meio da linguagem que a ciência utiliza para representar o mundo. Tendo a escola a responsabilidade de iniciar esse processo de alfabetização, podemos dizer que a educação escolar possui um papel significativo na apropriação da nossa cultura científica. Sforni (2004) afirma que o ensino é uma condição necessária para a aquisição de instrumentos cognitivos, os quais permitem o trânsito consciente na sociedade. Essa apropriação cognitiva, que também é cultural, necessariamente passa pela compreensão 
dos fenômenos físicos, químicos e biológicos. Estes, embora muitas vezes acabem passando por despercebidos, estão presentes no cotidiano de qualquer pessoa.

O não conhecimento, ou o entendimento incorreto/incompleto pelos estudantes dos conceitos químicos, e em particular os físico-químicos, tem sido apontado como um grande desafio para os professores e pesquisadores da área. Os estudos de Trejo \& Flores (2002) chamam a atenção para a existência de falsas idéias em torno de alguns conceitos termodinâmicos, como equilíbrio, espontaneidade e reversibilidade, que estão relacionadas às experiências pessoais que os alunos trazem.

Os estudos conduzidos por Gilbert et al. (2003) analisam a problemática do processo de ensino-aprendizagem de Química sob os aspectos histórico e filosófico, prático-experimental, de formação do currículo, das abordagens de ensino, dentre outros. Quanto a esse último, observa-se uma grande quantidade de pesquisas que abordam a formação de conceitos científicos em química e buscam maneiras de despertar o interesse dos estudantes, promovendo a aprendizagem com base no envolvimento ativo e na valorização das suas concepções alternativas. Da mesma forma, diversos estudos (Costa, 2008; Greca e Santos, 2005; Justil \& Driel, 2005) sugerem o uso da modelagem computacional no ensino de química como estratégia didática viável para a melhoria da compreensão de conceitos científicos em ambientes informatizados. Greca e Santos (2005) esclarecem ainda que diferentemente da Física, que utiliza principalmente a modelação para formalizar modelos matemáticos que descrevam sua realidade, na Química, deseja-se que os estudantes sejam capazes de modelar uma situação a partir da utilização de uma representação pictórica, que lhes permita raciocinar e expressar sua contrapartida simbólica.

Nessa pesquisa, pretendemos discutir alguns estudos relacionados à Teoria Sócio-histórica que possuem contribuição significativa para o campo educacional, no âmbito da Informática Educativa. Para isso, foram analisados os pressupostos e desdobramentos da teoria de Vygotsky, através da leitura de alguns autores neovygotskyanos como Davidov \& Zinchenko, Ivic, Daniels e Tudge. No que diz respeito à Educação Química, serão apresentadas algumas investigações (Costa, 2000; Gasparin, 2007; Mortimer, 2000; Sforni, 2004) voltadas à compreensão do processo de formação de conceitos, tomando-se como base as inter-relações entre os conhecimentos científicos e os de senso comum. Sendo assim, abordaremos diferentes concepções do processo de formação dos conceitos científicos, os quais servirão como base para a nossa proposta de pesquisa: a análise da construção de conceitos científicos relativos ao tema "reversibilidade termodinâmica", em episódios de ensino mediados pelo computador.

\section{Algumas contribuições da teoria Socio-Historica para a educação}

Conforme o referencial Marxista da época, Vygotsky procura explicar as características humanas a partir das relações sociais, ou seja, do convívio com outros indivíduos da espécie humana, capazes de elaborar cultura e fazer história. A atividade humana, para Vygotsky, possui três aspectos fundamentais que são: a) o fato de ser orientada por um objetivo; b) de fazer uso de instrumentos de mediação; e c) de produzir algo que se constitui como elemento da cultura, seja caracterizado por uma existência física ou simbólica, e que consiste na objetivação do ser humano (Zanella, 2007). O caráter mediado da atividade é crucial na obra de Vygotsky, por considerar a atividade instrumental sob uma perspectiva dialética em que indivíduo e meio físico se relacionam reciprocamente. Esses instrumentos mediadores (ferramentas e signos), por sua vez, são produtos da atividade social humana e são historicamente construídos. 
Outro aspecto importante presente na obra de Vygotsky (2001) diz respeito à relação temporal entre aprendizagem e desenvolvimento. Seus estudos mostraram que a aprendizagem está à frente do desenvolvimento, uma vez que as crianças adquirem certos hábitos e habilidades numa área específica antes de aprender a aplicá-los de modo consistente e arbitrário. Dessa forma, sendo a aprendizagem a força propulsora do desenvolvimento intelectual, não seria imprescindível que a criança alcançasse um determinado nível de desenvolvimento antes que se iniciasse a educação. De fato, a análise das considerações entre desenvolvimento e aprendizagem traçadas por Vygotsky levaram Ivic (1989) a fazer a seguinte afirmação:

"en un proceso natural de desarrollo, el aprendizaje se presenta como un medio que fortalece este proceso natural, pone a su disposición los instrumentos creados por la cultura que amplían la posibilidades naturales del individuo y reestructuran sus funciones mentales". (Ivic, 1989, p.462)

Na opinião de Davidov \& Zinchenko (1999), a teoria de Vygotsky pode dar uma grande contribuição à educação, sobretudo no que diz respeito aos conhecimentos práticos pedagógicos. A partir dela, podem ser construídas práticas de educação enfatizando o desenvolvimento de atividades que possibilitem a reprodução de necessidades e aptidões desenvolvidas socialmente. A educação e as práticas educativas, na concepção desses últimos, são justamente os meios sociais que, organizados, podem promover o desenvolvimento mental da criança. Entretanto, para que isso ocorra, devem se estabelecer condições propícias a vários tipos de atividades conjuntas, que posteriormente serão interiorizadas. A interiorização consiste na apropriação pelo sujeito das conquistas e conhecimentos historicamente produzidos, os quais ocorrem através das relações sociais que possibilitam ao sujeito construir-se como ser consciente (Zanella, 2007). Nesse sentido se iniciaram as pesquisas de cunho educacional, motivadas a explorar o conceito de zona de desenvolvimento próximo (ZDP) na tentativa de se compreender o processo de desenvolvimento da aprendizagem social e participativa. A zona de desenvolvimento próximo foi definida por Vygotsky como:

"a distância entre o nível de desenvolvimento real, determinado pela resolução independente de problemas, e o nível superior de desenvolvimento potencial, determinado pela resolução de problemas sob a orientação de um adulto ou em colaboração com pares mais capazes" (Vygotsky, 2001).

No seu livro, Daniels (2003) discute as diversas interpretações que foram tomadas a partir da definição de ZDP fornecida por Vygotsky. Nele, são expostas as considerações de Moll (1990), que atribui as mudanças de ZDP à criação, ao desenvolvimento e à comunicação de significado pelo uso colaborativo de meios mediacionais, e não na transferência de habilidades do parceiro mais capaz para o menos capaz. Outra discussão levantada diz respeito à aplicação do conceito de ZDP relativamente aos cenários. Há quem considere estritamente os cenários binários, em que possam existir somente um perito e um novato; e há os que considerem o "outro social" interagindo com esse indivíduo.

Uma questão que merece ser analisada diz respeito às pesquisas envolvendo atividades colaborativas, em grupos ou em pares. Nos seus estudos, Daniels (2003) chama a atenção para a existência de alguns aspectos inesperados dessa abordagem, 
alertando que em alguns casos pode haver efeitos negativos na aprendizagem. Em uma de suas pesquisas, Tudge (1993) teve o objetivo de analisar o efeito da colaboração em pares para a ZDP das crianças, enquanto elas resolviam um problema de equilíbrio dos pratos de uma balança. Seus resultados mostraram uma grande incidência de regressão nas crianças, exceto entre as de nível mais baixo, uma vez que elas passaram a utilizar regras menos complexas após as atividades em duplas. Ao que parece, somente as crianças que apresentaram um nível mais baixo de complexidade na resolução das atividades devem ter sido beneficiadas pelas interações. $O$ autor segue concluindo que a colaboração em pares pode provocar tanto o desenvolvimento, quanto a regressão na complexidade das regras que os aprendizes utilizam na resolução de problemas. Entretanto, um fator importante a ser considerado com vistas a esses resultados, diz ele, refere-se ao grau de segurança que cada criança apresenta ao utilizar as regras durante a interação, o que faz com que a ela possa regredir na complexidade da regra utilizada ao resolver o problema. Por fim, sugere-se que os efeitos da colaboração entre pares para o desenvolvimento cognitivo não sejam tão nítidos quanto supõem alguns pesquisadores.

Em outro estudo realizado por Tudge e colaboradores (1996) envolvendo crianças na resolução de tarefas matemáticas em duplas, foi constatado que as crianças cujo parceiro exibia raciocínio de alto nível tinham mais probabilidade de se beneficiar nas interações do que as crianças cujo parceiro não exibiam este tipo de raciocínio. Alguns aspectos negativos dessa abordagem citados pelos autores dizem respeito: i) ao fato de que simplesmente a disposição das crianças em duplas não causaria necessariamente avanços cognitivos; ii) o oferecimento de situações e questões que estejam potencialmente na zona de uma criança pode não ser suficiente para promover o seu desenvolvimento; e iii) ter um parceiro pode provocar distrações. Contudo, os autores concluem que ter um parceiro mais capaz traz mais benefícios do que trabalhar sozinho sem ter um feedback de um professor adulto.

Em uma pesquisa anterior (Costa \& Passerino, 2008), tivemos o objetivo de avaliar as atividades em pares realizadas por um grupo de estudantes licenciandos em Química. Nesse estudo, buscou-se promover um ambiente colaborativo e cooperativo entre os estudantes com o uso do software Modellus para verificar as modificações na ZDP dos alunos proporcionadas por interações sociais no ambiente computacional. Durante as atividades, os alunos foram distribuídos em pares com o seguinte cuidado: aqueles que apresentaram baixo desempenho formaram par com os que apresentaram maior desempenho no pré-teste. Ao término das atividades, realizou-se um pós-tese para medir o desempenho dos alunos e a contribuição dos colegas nas interações mediadas pelo professor naquele ambiente. Nossos estudos mostraram uma melhora no desempenho dos alunos em função das atividades computacionais realizadas sob forma colaborativa e com o feedback do professor. Vale ressaltar que a cooperação e a colaboração funcionaram como mecanismos sócio-cognitivos favoráveis ao processo de ensino-aprendizagem, tendo em vista as modificações ocorridas na ZDP desses alunos, principalmente os que obtiveram menor rendimento no pré-teste. Destacamos também a importância na atuação do professor no planejamento e na execução da proposta pedagógica, além de sua participação como observador e mediador do processo.

Levando em conta tais pesquisas, podemos afirmar que as atividades colaborativas e cooperativas realizadas em duplas podem trazer benefícios, principalmente para os alunos que apresentam rendimento inicial mais baixo ou são menos experientes. Porém, entendemos que as conclusões tomadas com base na proposição desse tipo de estudo devem ser cuidadosamente analisadas em termos de processos de interação entre os sujeitos, sob o risco de se tirar conclusões parciais, tendo em vista a complexidade da questão.

V. $7 \mathrm{~N}^{\mathrm{o}}$ 3, dezembro, 2009 


\section{Processo de Formação de conceitos científicos}

Vygotsky afirma que os conceitos científicos são aqueles introduzidos por um professor na escola. Eles são caracterizados por alto grau de generalidade e por sua relação com os objetos, mediada por outros conceitos. Por outro lado, os conceitos cotidianos ou espontâneos são adquiridos pela criança fora de contextos em que a instrução esteja explícita. Entretanto, alguns autores como Van der Veer (1998) e Bernstein (1999) preferem discutir essa questão numa abordagem histórica para justificar seus pontos de vista acerca do papel da escola na formação dos conceitos científicos. Na concepção desses últimos, convêm considerar que os conhecimentos científicos podem ser desenvolvidos também fora das instituições formais de escolarização.

Os episódios de ensino que se apóiam nos estudos de Davidov (1988) devem enfatizar a aquisição do conhecimento teórico em detrimento ao conhecimento empírico. Nesse caso, para que o processo de formação de conceitos científicos seja eficaz, é necessário que se promova a tomada de consciência da forma e da estrutura conceitual. Sendo assim, convém considerar o acesso individual aos conceitos científicos, o controle entre eles, e da mesma forma promover a interação entre esses últimos e os conceitos cotidianos. A conexão entre os conceitos espontâneos, que surgem pela aprendizagem empírica, e os conceitos científicos, que se desenvolvem pelo conhecimento teórico, é vista como a principal dimensão da ZDP. Quando se trabalha segundo essa abordagem, os professores devem tentar explicar leis gerais, propondo que os alunos investiguem e apliquem essas leis em alguns casos de forma a incorporar conceitos mais específicos.

No Brasil, são muitos os pesquisadores da área de Educação Química (Costa, 2000; Gasparin, 2007; Sforni, 2004; Mortimer, 2000) que têm estudado o processo de formação de conceitos científicos. Costa (2000) se apóia na teoria de Bachelard ${ }^{1}$ para propor práticas pedagógicas que se utilizem de atividades-problema, criando dificuldades para que se atinja o conhecimento científico. Ele discute o papel da experimentação para a construção de conceitos, destacando que, em novas experiências, o conceito primitivo deve ir se deformando e incorporando novas condições de aplicação. Além disso, afirma que a série de aproximações sucessivas nas aplicações leva ao aperfeiçoamento dos conceitos, de modo que os conceitos científicos passam a ser constituídos pelo agrupamento das aproximações sucessivas realizadas de forma ordenada.

Gasparin (2007) discute a construção dos conceitos científicos com base em elementos da Teoria Histórico-cultural. Ele assume que o ensino e a aprendizagem trazem consigo toda a história de sua constituição e que operam de um modo específico no processo de construção e transmissão do conhecimento. Sua base são as interações sociais que formam as funções psicológicas superiores resultantes de um processo histórico e social. Nessa concepção, a criança se apropriaria ainda na idade pré-escolar de diferentes conceitos, mas na escola ela teria acesso ao saber científico sistematizado, adquirindo novos conteúdos e principalmente formas mais elevadas de pensamento. No processo escolar ela articula novos e velhos conhecimentos, ou seja, une seus conceitos cotidianos aos científicos, pela mediação do professor, estabelecendo novas relações, o que lhe permite ir muito além do imediatamente perceptível.

Na opinião de Sforni (2004), ao buscarmos a especificidade dos conceitos científicos, é fundamental percebermos que o seu conteúdo comporta níveis de

\footnotetext{
1 Autor da "filosofia do não", ele propõe uma ruptura das verdades encontradas no conhecimento tradicional.

V. $7 \mathrm{~N}^{\mathrm{o}}$ 3, dezembro, 2009
} 
organização do pensamento que não se limitam a captar apenas o aspecto empírico, externo ou observável dos objetos e fenômenos. E por isso mesmo, eles diferenciam-se dos conceitos espontâneos também na forma de sua apropriação e re-elaboração. A autora enfatiza que essa apropriação a qual nos referimos não é um processo simples e por isso exige situações mediadas intencionalmente. Para tanto, propõe situações de ensino fundamentadas na Teoria da Atividade $^{2}$ para oferecer elementos que contribuam na aprendizagem de conceitos científicos e na organização do ensino.

As pesquisas conduzidas por Mortimer (Mortimer, 2000; Amaral e Mortimer, 2006) baseiam-se na concepção de que a aprendizagem ocorre através do envolvimento ativo dos aprendizes e na valorização dos seus conhecimentos prévios. Ele não assume a aprendizagem formal como uma mudança dos conceitos pelos alunos, mas como uma mudança de perfil conceitual do estudante, em que o novo perfil inclui também (mas não exclusivamente) as novas idéias científicas. Diferentes idéias sobre um mesmo conceito poderiam co-existir em uma mesma pessoa, e poderiam ser usadas em diferentes contextos, de acordo com sua necessidade ou conveniência.

A existência desse perfil pluralista permite criar zonas de perfil conceitual que se constituem em diferentes pontos de vista em relação a determinado conceito específico. Amaral e Mortimer (2006) afirmam que essas zonas podem, em princípio, ser compartilhadas por todos os indivíduos, mas somente alguns deles exibirão todas elas. Desta forma, a ampliação das zonas em determinado perfil individual subentende uma compreensão mais completa da realidade.

Com base no exposto, acreditamos que o estabelecimento de uma relação positiva entre a evolução do perfil individual dos estudantes durante os episódios de ensino e a evolução do perfil dos grupos pode indicar o alargamento das suas ZDP's. É nesse sentido que estamos propondo estratégias de ensino baseadas na mudança das zonas de perfil conceitual dos estudantes envolvendo a formação de conceitos relativos à reversibilidade termodinâmica nos episódios de ensino mediados pelo computador.

\section{Estruturação dos episódios de ensino em termodinâmica}

Mortimer (2000) define episódios de ensino como "unidades de análise dos processos de elaboração microgenética em sala de aula". Esses episódios compreendem um conjunto de enunciados que criam um contexto para a emergência de significados em sala de aula. Considerando que podem existir diferentes visões sobre os conceitos dependendo do contexto em que o indivíduo se encontra, apresentaremos uma proposta para estruturação de episódios de ensino no ambiente computacional que tem por objetivo ampliar as zonas dos perfis conceituais dos estudantes. Essas modificações nas zonas de perfil conceitual caracterizariam uma compreensão mais completa das transformações e conceitos físico-químicos, planejada por meio do desenvolvimento de atividades mediadas em sala de aula, em um período relativamente curto de tempo.

Os aspectos da estrutura de análise compreenderão quatro pontos: intenções do professor, conteúdo, abordagem comunicativa e padrões de interação. O primeiro relaciona-se com os objetivos propostos e pode variar ao longo das atividades de aula; o segundo corresponde às categorias de abordagens, que podem ser descritivas, explicativas ou de generalização; o terceiro aspecto corresponde ao tipo de comunicação entre professor e alunos; e o quarto diz respeito aos padrões existentes nas interações discursivas entre professores e alunos na sala de aula.

\footnotetext{
${ }^{2}$ Teoria desenvolvida por Leont'ev para explicar os processos psicológicos baseados na ação mediada do sujeito.

V. $7 \mathrm{~N}^{\mathrm{o}}$ 3, dezembro, 2009
} 
Com relação aos aspectos anteriormente citados, pode-se afirmar que as intenções do professor serão: checar as idéias dos alunos e criar situações apoiadas no(s) texto (s) sugerido (s) que levarão ao questionamento das concepções prévias dos alunos, além de conduzir as atividades de modelagem computacional. Quanto ao conteúdo, este poderá levar em conta as três formas de abordagem: descritiva, explicativa e de generalização. O tipo de comunicação professor-alunos utilizará basicamente duas abordagens: interativa dialógica e interativa de autoridade. Quando o professor estiver interessado em explorar novas idéias e quiser fazer com que os alunos reflitam sobre o tema levantado e exponham seus pontos de vista, será utilizada a abordagem interativa dialógica. Por outro lado, nos momentos em que se desejar transmitir um conhecimento em que o único ponto de vista - o científico - seja considerado, será usada a abordagem interativa de autoridade. Em relação aos padrões de interação, serão promovidas as interações não triádicas em cadeia aberta, em oposição às interações de três partes, onde a simetria de interação é atingida logo no primeiro retorno (iniciação-retorno-avaliação). Nesse caso, o professor apresentará um feedback $(\mathrm{F})$ ou um prosseguimento $(\mathrm{P})$ à fala do aluno, visando dar prosseguimento ao processo de interação conduzido pela iniciação (I) e posterior resposta (R). Diz-se que a cadeia é aberta porque normalmente o professor não apresenta um processo de avaliação ao final da seqüência.

A partir do conjunto de informações coletadas por meio de entrevistas e gravações em vídeo das aulas, tentaremos identificar as mudanças nas zonas dos perfis conceituais dos alunos em relação aos conceitos relativos à reversibilidade termodinâmica. Nesse sentido, inicialmente serão determinadas as categorias que constituirão essas zonas do perfil conceitual com base nos conhecimentos apresentados pelos estudantes. A zona perceptivo-indutiva (ZP/I) corresponde às idéias que surgem das impressões imediatas, das sensações e intuições, que não são relativizadas pela consideração das condições em que o processo ocorre (Amaral e Mortimer, 2006). Sob essa perspectiva, o conceito de reversibilidade normalmente está associado aos processos que podem ocorrer tanto em um sentido quanto em outro. A zona empírica (ZE) refere-se às idéias que levam em consideração as condições termodinâmicas do sistema, como temperatura e pressão, ou carregam expressões que se julga estarem relacionadas a essas condições. Na zona formalista (ZF) devem estar presentes as relações matemáticas puras - como as expressões de trabalho reversível e irreversível sem que estas estejam integradas aos conhecimentos conceituais. Já a zona racionalista (ZR) compreende conhecimentos integrados de significados conceituais e de formalismos, sendo por isso considerada de maior complexidade cognitiva. Para estar associado a esta zona, o conceito de reversibilidade deve ser pensado como uma condição possível dos sistemas, analisada sob os aspectos macro e microscópicos, não apenas de um sistema isolado, mas a partir de uma visão universal.

Quanto aos instrumentos de procedimento e análise, serão desenvolvidos além dos episódios de ensino, os mapas de atividades para a caracterização do contexto da sala de aula. Esses mapas correspondem a tabelas em que são contemplados alguns campos de preenchimento como: o tempo estipulado das atividades, sua descrição, os principais temas, as ações dos participantes e os comentários do professor. Um exemplo de mapa de atividade pode ser encontrado em Amaral e Mortimer (2006, p. 267). Eles sugerem a utilização desses mapas por julgarem o seu uso importante para uma melhor compreensão e contextualização dos enunciados. Neste trabalho, sua importância devese à facilidade de sistematização das informações que deverão compor o material para posterior análise.

Uma vez definidos os instrumentos analíticos, serão estruturados os episódios de ensino com base na abordagem de conceitos relacionados ao Primeiro Princípio da 
Termodinâmica aplicado aos sistemas gasosos, com foco nas transformações reversíveis e irreversíveis. A Tabela 1 apresenta os episódios de ensino sugeridos para a abordagem do assunto, bem como a sistematização dos aspectos epistemológicos e discursivos em que estão contemplados nesses episódios.

Tabela 1: Síntese analítica dos episódios de ensino realizados na sequiência de aulas.

\begin{tabular}{|c|c|c|c|c|c|}
\hline $\begin{array}{l}\text { Episódios, } \\
\text { turnos e } \\
\text { sujeitos }\end{array}$ & $\begin{array}{l}\text { Zonas } \\
\text { do } \\
\text { perfil }^{3}\end{array}$ & $\begin{array}{l}\text { Abordagem } \\
\text { do conteúdo }\end{array}$ & $\begin{array}{l}\text { es do } \\
\text { or }\end{array}$ & em & $\begin{array}{l}\text { Padrões } \\
\text { de } \\
\text { interação }\end{array}$ \\
\hline \multicolumn{6}{|c|}{$\begin{array}{l}\text { Episódio 1.1: Caracterização dos sistemas físico-químicos e introdução aos conceitos } \\
\text { de energia interna, calor e trabalho }\end{array}$} \\
\hline $\begin{array}{l}\text { Segmento1: } \\
\text { Professor }(\mathrm{P}) \\
\text { e alunos }(\mathrm{A})\end{array}$ & $\begin{array}{l}\mathrm{ZP} / \mathrm{I}- \\
\mathrm{ZE}\end{array}$ & $\begin{array}{l}\text { Generalização } \\
\text { empírica e } \\
\text { teórica }\end{array}$ & $\begin{array}{l}\text { Checar o } \\
\text { entendimento } \\
\text { dos alunos }\end{array}$ & & $\begin{array}{l}\text { Iniciação - } \\
\mathrm{A}_{1}-\mathrm{A}_{2}-\ldots\end{array}$ \\
\hline $\begin{array}{l}\text { Segmento 2: } \\
\text { Professor }(\mathrm{P})\end{array}$ & ZR & $\begin{array}{l}\text { Exp } \\
\text { gen } \\
\text { teór }\end{array}$ & as & & lção - \\
\hline \multicolumn{6}{|c|}{$\begin{array}{l}\text { Episódio 1.2: Discussão do conceito de reversibilidade segundo duas óticas: a do } \\
\text { livro-texto e a do pensamento de Ylya Prigogine }{ }^{5}\end{array}$} \\
\hline $\begin{array}{l}\text { Segmento 1: } \\
\text { Alunos (A) }\end{array}$ & ZR & $\begin{array}{l}\text { Generalização } \\
\text { teórica }\end{array}$ & $\begin{array}{l}\text { Discussão entre } \\
\text { os alunos }\end{array}$ & & $\begin{array}{l}\mathrm{A}_{1}-\mathrm{A}_{2}- \\
\ldots\end{array}$ \\
\hline $\begin{array}{l}\text { ento 2: } \\
\text { sor (P) } \\
\text { Os (A) }\end{array}$ & ZR & $\begin{array}{l}\mathrm{Ge} \\
\text { teó }\end{array}$ & $\begin{array}{l}\text { Che } \\
\text { ent } \\
\text { dos }\end{array}$ & $\mathrm{d}$ & $\begin{array}{l}\text { ação - } \\
\mathrm{A}_{2}-\ldots\end{array}$ \\
\hline \multicolumn{6}{|c|}{$\begin{array}{l}\text { Episódio 2.1: Cálculo de trabalhos reversíveis e irreversíveis em transformações } \\
\text { isotérmicas de gases }\end{array}$} \\
\hline $\begin{array}{l}\text { Segmento 1: } \\
\text { Alunos (A) }\end{array}$ & $\begin{array}{l}\mathrm{ZE}-\mathrm{ZF} \\
-\mathrm{ZR}\end{array}$ & $\begin{array}{l}\text { Explicação e } \\
\text { generalização } \\
\text { teórica }\end{array}$ & $\begin{array}{l}\text { Aplicar as } \\
\text { idéias } \\
\text { científicas }\end{array}$ & $\begin{array}{l}\text { Interativa de } \\
\text { autoridade }\end{array}$ & $\begin{array}{l}\text { Iniciação - } \\
\mathrm{A}_{1}-\mathrm{P}-\ldots\end{array}$ \\
\hline \multicolumn{6}{|c|}{$\begin{array}{l}\text { Episódio 2.2: Realização da atividade de modelagem computacional com o Modellus } \\
\text { para o desenvolvimento de equações de trabalhos isotérmicos reversíveis e } \\
\text { irreversíveis }\end{array}$} \\
\hline $\begin{array}{l}\text { Segmento 1: } \\
\text { Professor }(\mathrm{P}) \\
\text { e alunos }(\mathrm{A})\end{array}$ & $\begin{array}{l}\mathrm{ZE}-\mathrm{ZF} \\
-\mathrm{ZR}\end{array}$ & $\begin{array}{l}\text { Descrição, } \\
\text { explicação e } \\
\text { generalização } \\
\text { teórica }\end{array}$ & $\begin{array}{l}\text { Ilustrar as } \\
\text { idéias } \\
\text { científicas }\end{array}$ & $\begin{array}{l}\text { Interativa } \\
\text { dialógica }\end{array}$ & $\begin{array}{l}A_{1}-A_{2} \\
A_{3}-A_{4} \\
\cdots\end{array}$ \\
\hline
\end{tabular}

Cada segmento dos episódios de ensino apresentados favorecerá uma ou mais zonas do perfil conceitual. Espera-se conseguir observar as mudanças que ocorrerão nessas zonas, através dos discursos dos alunos e da realização das suas atividades. Os episódios de ensino serão realizados em grupos ou em duplas, com o propósito de considerar o fator sócio-cultural na formação dos conceitos científicos a partir das experiências prévias dos alunos e das modificações associadas aos diferentes contextos

\footnotetext{
${ }^{3}$ Referem-se às principais zonas do perfil estimuladas nos episódios.

${ }^{4}$ Representam o padrão de interações esperado.

5 Pesquisador Russo ganhador do Prêmio Nobel de Química em 1977 pelos seus estudos em termodinâmica de processos irreversíveis com a formulação da teoria das estruturas dissipativas.

${ }^{6}$ Software de simulação e modelagem computacional disponível em: http://modellus.fct.unl.pt/

V. $7 \mathrm{~N}^{\circ}$ 3, dezembro, 2009
} 
que surgirão em sala de aula. Desta forma, pretendemos estabelecer uma relação entre a evolução nas zonas dos perfis individuais dos alunos e nas zonas dos perfis dos grupos, durante os episódios de ensino. A etapa experimental da nossa pesquisa consistirá na aplicação dessa proposta metodológica entre os estudantes de uma turma do curso de licenciatura em Química, durante o período regular de aula.

\section{Considerações Finais}

Nossa proposta metodológica foi construída com base em estudos relativos à Teoria Sócio-histórica, assumindo a aprendizagem como um processo social compartilhado e gerador de desenvolvimento. Dentre os autores que contribuíram com a nossa pesquisa, destacamos principalmente os estudos de Mortimer e Amaral (Mortimer, 2000; Amaral e Mortimer, 2006) sobre a existência das zonas de perfil conceitual e suas implicações pedagógicas. Nessa concepção, assume-se a existência de diferentes visões sobre um mesmo conceito científico, dependendo do contexto em que o indivíduo se encontra e de acordo com sua necessidade ou conveniência. Além disso, admite-se a aprendizagem formal não como uma mudança dos conceitos químicos pelos alunos, mas uma mudança de perfil conceitual do estudante, em que o novo perfil inclui também (mas não exclusivamente) as novas idéias científicas. Vislumbramos nesses estudos uma grande possibilidade de aplicação no ensino de conceitos físico-químicos, especialmente o de reversibilidade termodinâmica. Dessa forma, apresentamos uma proposta para estruturação de episódios de ensino em ambientes computacionais com o objetivo de ampliar as zonas dos perfis conceituais dos estudantes no tema escolhido.

\section{Referências Bibliográficas}

AMARAL, E. M. R. ; MORTIMER, E. F. . Uma metodologia para análise da dinâmica entre zonas de um perfil conceitual no discurso da sala de aula. In: Flávia Maria Teixeira dos Santos; Ileana Maria Greca. (Org.). A pesquisa em ensino de ciências no Brasil e suas metodologias. Unijuí: Editora Unijuí, 2007, p. 239-296.

BERNSTEIN, B. "Vertical and horizontal discourse: an essay". British Journal of Sociology of Education, 1999, 20,2. p. 157-173.

CHASSOT, A.I. Alfabetização científica: uma possibilidade para a inclusão social. Revista Brasileira de Educação, n. 22. Jan/Fev/Mar/Abr 2003.

COSTA, R. C.. Construção do conhecimento científico segundo algumas contribuições da epistemologia de Bachelard. In: Roque Moraes. (Org.). Construtivismo e ensino de ciências: reflexões epistemológicas e metodológicas. $1^{\text {a }}$ ed. Porto Alegre: EDPUCRS, 2000, v. 1, p. 69-101.

COSTA, R. G. ; PASSERINO, L. M. . Uma proposta pedagógica para o uso da modelagem computacional no curso de licenciatura em química do Cefet Campos. RENOTE. Revista Novas Tecnologias na Educação, v. 06, 2008, p. 01-10.

DANIELS, Harry. Vygotsky e a pedagogia. São Paulo: Loyola, 2003.

DAVIDOV, V. La Enseñanza Escolar y el Desarollo Psiquico: Investigación psicológica teórica y experimental. Moscou: Editorial Progresso, 1988.

DAVYDOV,V.V. \& ZINCHENKO,V.P. "A contribuição de Vygotsky para o desenvolvimento da psicologia" In: DANIELS, Harry (org.). Vygotsky em foco: pressupostos e desdobramentos. Campinas: Papirus, 1999. p.151-167. 
GASPARIN, J. L. A construção dos conceitos científicos em sala de aula (no prelo). In: Nádia Lúcia Nardi (Org.). Educação: Visão Crítica e Perspectivas de Mudanças. $1^{\circ}$ ed. Concórdia - SC: Editora da Universidade do Contestado, 2007, v. 1, p. 1-25.

GRECA, Ileana M.; SANTOS, Flávia M. T. dos. Dificuldades da generalização das estratégias de modelagem em ciências: o caso da física e da química. Investigações em Ensino de Ciências. 2005, V10 (1), pp. 31-46.

GILBERT, John K.; TREAGUST, David F.; JUSTI, Rosaria; JONG, Onno de; VAN DRIEL, Jan H. Chemical Education: Towards Research-Based Practice, Vol. 17. December 2003, 452pp.

JUSTIL, Rosária; DRIEL, Jan van. A Case Study of the Development of a Beginning Chemistry Teacher's Knowledge about Models and Modelling. Research in Science Education, 2005. p. 197-219.

MOLL, L. Vygotsky and education: Interactional Implications and applications of sociohistorical psychology, 1990. In: DANIELS, Harry. Vygotsky e a pedagogia. São Paulo: Loyola, 2003.

MORTIMER, E. F. Linguagem e Formação de Conceitos no Ensino de Ciências. $1^{\text {a }}$ ed. Belo Horizonte: Editora UFMG, 2000. v. 01. 383 p.

SFORNI, Marta Sueli de Faria. Aprendizagem conceitual e organização do ensino: contribuições da teoria da atividade. Araraquara: JM Editora, 2004. 200p.

TREJO, L. M., FLORES, S. The importance os students' misconceptions on learning thermodynamics. In: VI Conferência Ibero americana sobre Equilíbrio de Fases para o Projeto de Processos (EQUIFASE 2002), Foz do Iguaçu, Brasil, Anais da VI Conferência Ibero-Americana Sobre Equilíbrio de Fases para o Projeto de Processos, 2002.

TUDGE, J., Vygotsky, la zona de desarrollo próximo y la colaboración entre pares: connotaciones para la práctica del aula. In: MOLL, L. C. Vygotsky y la Educación. Buenos Aires: Aique, 1993. pp-187-207.

TUDGE, J. R. H., Winterhoff, P., Hogan, D. M. "The cognitive consequences of collaborative problem solving with and without feedback", Child Development, 67, 1996. p. 2892-2909.

VAN DER VEER, R.. "From concept attainment to knowledge formation". Mind, Culture and Activity 5 (2), 1998, p. 89-94.

VYGOTSKY, L. S. A Construção do Pensamento e da Linguagem. Tradução Paulo Bezerra, $1^{\circ}$ ed. São Paulo: Martins Fontes, 2001.

ZANELLA, A. V. . Vygotski: contexto, contribuições à psicologia e o conceito de zona de desenvolvimento proximal. $1^{a}$ ed. Itajaí: Editora da UNIVALI, 2007. v. único. $129 \mathrm{p}$. 